\title{
Defect mediated manipulation of nanoclusters on an insulator
}

\section{SUBJECT AREAS: \\ SURFACES, INTERFACES AND THIN FILMS}

ELECTRONIC STRUCTURE

DESIGN, SYNTHESIS AND PROCESSING

NANOPARTICLES

Received

23 October 2012

Accepted

24 January 2013

Published

14 February 2013

\section{Correspondence and} requests for materials should be addressed to T.H. (teemu.hynninen@ aalto.fi) or C.B. (barth@cinam.univmrs.fr)

\author{
Teemu Hynninen ${ }^{1,2}$, Gregory Cabailh ${ }^{3,4}$, Adam S. Foster ${ }^{1,2} \&$ Clemens Barth ${ }^{3,4}$
}

\author{
${ }^{1}$ COMP Centre of Excellence, Department of Applied Physics, Aalto University, P.O. Box 11 100, Fl-00076 Aalto, Finland, \\ ${ }^{2}$ Department of Physics, Tampere University of Technology, P.O. Box 692, Fl-33 101 Tampere, Finland, ${ }^{3} \mathrm{CNRS}$, UMR7325, 13288, \\ Marseille, France, ${ }^{4}$ Aix-Marseille University, CINaM, 13288, Marseille, France.
}

With modern scanning probe microscopes, it is possible to manipulate surface structures even at the atomic level. However, manipulation of nanoscale objects such as clusters is often more relevant and also more challenging due to the complicated interactions between the surface, cluster and apparatus. We demonstrate the manipulation of nanometer scale gold clusters on the $\mathrm{NaCl}(001)$ surface with a non-contact atomic force microscope, and show that the movement of clusters is in certain cases constrained to specific crystallographic directions. First principles calculations explain this kinetic anisotropy as the result of the cluster attaching to surface defects: cation vacancies allow the clusters to bond in such a way that they only move in one direction. Constraining the movement of clusters could be exploited in the construction of nanostructures or nanomechanical devices, and the manipulation signatures may also be used for identifying cluster-defect complexes.

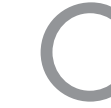
ontrolling structures at the atomic level is the ultimate challenge in nanofabrication - absolute control over the positions of individual atoms, molecules, or clusters on substrates allows for nearly complete freedom in material composition or in the fabrication of nanodevices. Although arranging structures atom-byatom is attractive from the perspective of fundamental interest in surface physics and chemistry, for many applications manipulation of larger units is more relevant. With respect to metallic clusters, interest stems from catalysis where clusters promote many catalytic reactions ${ }^{1-3}$, but also from nanotechnology where clusters can be used as building blocks for nanoelectronic devices ${ }^{4}$. In tribology, clusters can even serve as units for probing the friction at the nanoscale ${ }^{5,6}$. In all these domains, manipulating clusters as single objects enables the systematic study of the impact of the specific and voluntarily chosen adsorption site on the physical, mechanical, electronic and chemical properties of a single cluster ${ }^{7}$. While manipulation of atoms was achieved already in 1990 with scanning tunneling microscope $(\mathrm{STM})^{8}$, atomic manipulation with non-contact atomic force microscopy (nc$\mathrm{AFM})^{9}$ has been a much more recent development ${ }^{10}$. In particular, manipulation on insulating surfaces remains particularly challenging ${ }^{11,12}$, yet insulators are a crucial class of materials in nanoelectronics and nanocatalysis.

Similar to STM ${ }^{13}$, two methods are used to manipulate clusters by nc-AFM: the first method consists of moving the cluster by pushing during a spectroscopy step (Fig. 1 (a)). Here, the AFM tip is brought down vertically near the cluster until at some point the repulsive interaction between the tip and the cluster is strong enough to make the cluster move. In the second method the tip is scanned above the cluster (Fig. 1 (b)), preferentially in the constant height AFM mode ${ }^{14-16}$. At a specific distance, as the tip approaches the cluster laterally, a force between both moves the cluster. For simplicity, we will call the former method kicking and the latter sliding.

This work investigates methods for directing the manipulation of a nanocluster beyond the constraint of the tip's scanning direction. In particular we demonstrate the possibility of using intrinsic defects on the surface to mediate the diffusive behaviour of the clusters. We have chosen the $\mathrm{NaCl}(001)$ surface as a model insulator substrate firstly because it can be easily prepared and examined by nc-AFM even at the atomic scale ${ }^{17}$, but also because the characteristic defects at it's surface are well-established ${ }^{18}$. Furthermore, $\mathrm{NaCl}$ and other alkali halide surfaces have been key in breakthrough nc-AFM studies in atomic ${ }^{19-21}$ and charge identification ${ }^{18,22}$, as well as providing prototype substrates for the study of adsorbed molecules ${ }^{23-25}$, self-assembly ${ }^{26}$, friction ${ }^{27,28}$, and local electronic structure ${ }^{29,30}$.

\section{Results}

Manipulation on terraces. When single neutral gold atoms are deposited on perfect $\mathrm{NaCl}(001)$ surfaces, the gold atoms diffuse on the terraces due to their high mobility at room temperature ${ }^{31-33}$ until they reach a step-edge, where they aggregate into clusters ${ }^{1,34}$. At room temperature and low gold coverage, the steps are decorated by gold 


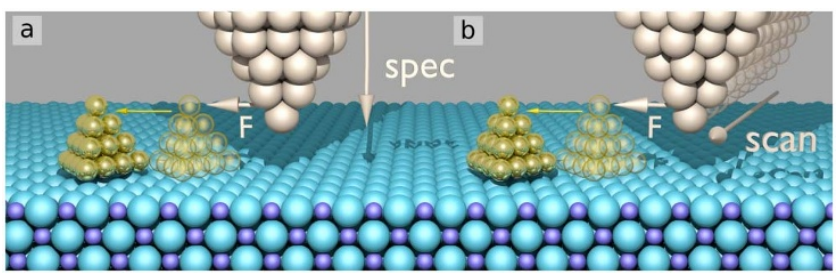

Figure $1 \mid$ Principles used in the manipulation of clusters. (a) the cluster is moved by kicking during a spectroscopy step; (b) moving the cluster by sliding during scanning.

clusters whereas on the flat terraces very few clusters can be found because of the lack of surface defects that would act as nucleation sites (see further discussion in the Supplementary information). The image in Fig. 2 (a) exemplifies a typical decoration of steps (along $\langle 100\rangle$ equivalent directions) by gold clusters. The clusters have a mean diameter of $5 \mathrm{~nm}$ ( $\sim 2500$ atoms), which was estimated from the flux of incoming gold during the deposition.

In this first series of manipulation experiments, we focus on one of the rare clusters on the terrace (highlighted in green in Fig. 2) and consider its manipulation characteristics. The cluster was first imaged with a high magnification in the constant height mode (Fig. 2 (b)). In the figures, the slow scanning direction of the AFM tip is always from the bottom to the top. The same cluster was imaged several times while gradually bringing the tip closer to the surface. At a specific tip-surface distance the cluster suddenly disappeared during imaging (arrow in Fig. 2 (e)) by escaping behind the tip to the region that had just been imaged to the position shown in Fig. 2 (c). This kind of backheel led to a $16 \mathrm{~nm}$ long movement of the cluster along the [100] direction. In a second manipulation step the same cluster was imaged again and the tip-surface distance was further reduced. Now, the cluster could be moved by sliding in front of the tip along the [110] direction (Fig. 2 (f)). Because the cluster is moved in front of the tip from line to line, it appears with an elongated contrast, reflecting its movement. The new position of the cluster, which is $11 \mathrm{~nm}$ further away from the old one, can be seen in Fig. 2 (d).

Manipulation from step-edges. In Fig. 3, similar clusters of about 2000 atoms decorate intersecting $\langle 110\rangle$ and $\langle 100\rangle$ steps on the $\mathrm{NaCl}(001)$ surface. One of the clusters is highlighted (I in Fig. 3 (a), green color) at the $\langle 110\rangle$ step-edge. After recording a series of high resolution images at this site, the last image of this series shows that the cluster was moved $12 \pm 1 \mathrm{~nm}$ from the step (to position II in Fig. 3 (b)). We continued to move the same cluster, now on the terrace, by sliding about $15 \pm 1 \mathrm{~nm}$ along the $\langle 110\rangle$ direction (Fig. 3 (c)), which has been verified in a following image with a larger scale (Fig. 3 (d)). We could move the cluster also by kicking with a vertically approaching tip in a spectroscopy step. Figure 3 (e) shows that in this case the cluster could also be moved by $16 \pm 1 \mathrm{~nm}$ from its initial position. Altogether, the cluster was moved by the tip dozens of times during the whole manipulation process. Regardless of the method of manipulation, the events always resulted in cluster movement along equivalent $\langle 110\rangle$ directions on the surface (see path of cluster in Fig. 3 (f)). This extremely anisotropic movement is in clear contrast to the cluster found on the terrace, which could easily be moved in different directions.

Calculated manipulation kinetics. In order to understand the movement of clusters during manipulation and pinpoint the origin of the experimentally observed differences, we sample the possible pathways for moving 20 and 100 atom gold clusters (Fig. 4 (a)) using density functional calculations. With the smaller $\mathrm{Au}_{20}$ cluster we perform extensive analysis on the adsorption configurations and diffusion pathways while the larger $\mathrm{Au}_{100}$ cluster is used in selected cases to assess scaling with respect to cluster size.

$\mathrm{The} \mathrm{Au}_{20}$ cluster is found to adsorb on the perfect surface (adsorption energy $0.5 \mathrm{eV}$ ) and the energy barriers for moving the cluster in any given direction are very low, about $0.2 \mathrm{eV}$ (Fig. 4 (b)). At room temperature, such a diffusion barrier corresponds to a Boltzmann factor of $10^{-4}$, and thus such a small cluster should move spontaneously due to thermal energy. Furthermore, the barriers for moving the cluster in either $\langle 100\rangle$ or $\langle 110\rangle$ directions are practically the same and thus no direction should be preferred.

The calculated high mobility on the ideal terrace strongly implies the presence of a defect under the cluster in Fig. 2 - else the cluster would have diffused to a step during growth. Hence, we also consider the role of defects in cluster adsorption and diffusion. The calculations show that in the presence of surface vacancies, or F-centres, the cluster adsorbs even more strongly than on the perfect surface, as the adsorption energies are 2.1,2.5, and $1.1 \mathrm{eV}$ for adsorption at neutral $\mathrm{Cl}, \mathrm{Na}$ and double vacancies, respectively. (Defect charge state influence is discussed in the Supplementary information.) Also the diffusion energy barriers are much higher when defects are present, as discussed below, meaning it is much more difficult to move the cluster, and this verifies that defects can act as anchors for the clusters.

In the presence of a $\mathrm{Cl}$ vacancy, the barriers for moving the cluster are $0.8-1.1 \mathrm{eV}$ and the anisotropy is relatively weak (Fig. 4 (c)). A cluster bonded to a Na vacancy, on the other hand, prefers a [110] direction where the barrier is calculated to be $0.7 \mathrm{eV}$. In comparison, the $\langle 100\rangle$ barriers are $0.9 \mathrm{eV}$, and in the perpendicular [ 110$]$ direction the barrier is $1.2 \mathrm{eV}$ (Fig. 4 (d)). Similar calculations with a 100 atom gold cluster show that this is still the case even when the clusters grow. Figure 4 (e) shows the calculated barriers for the $\mathrm{Au}_{100}$ $+\mathrm{Na}$ vacancy complex. When the vacancy is located near the cluster edge, in the energetically favored position, the anisotropy is even stronger than for the small cluster (barriers $0.5-1.1 \mathrm{eV}$ ).
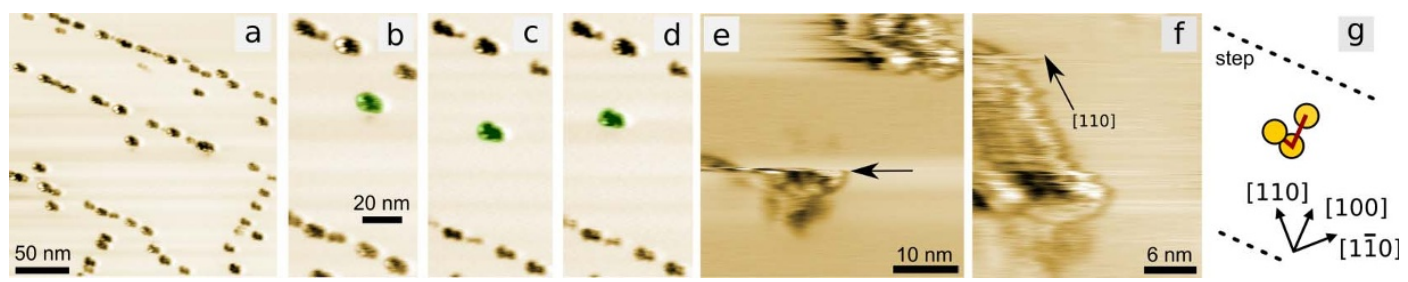

Figure $2 \mid$ Manipulation of a Au cluster (green) on $\mathrm{NaCl}(001)$ along the [110] and [100] directions (nominal Au thickness: 0.3 mono-layers, flux: $4.1 \times 10^{12}$ atoms $/ \mathrm{cm}^{2} / \mathrm{s}$ ). (a) The surface region before the manipulation (density: $0.9 \times 10^{11}$ clusters $/ \mathrm{cm}^{2}$, cluster-cluster mean distance at steps: $\sim 20 \mathrm{~nm}$ ). (b) - (d) The cluster before (b), after the first (c) and after the second (d) manipulation experiment. (e) During the first manipulation, a part of the cluster could be still imaged until the position labeled by the arrow where the cluster suddenly started to escape behind the tip (backheel). (f) During the second manipulation step, the cluster was moved by sliding along the [110] direction. (g) Schematic of the two manipulation steps.

(a) $250 \times 250 \mathrm{~nm}^{2}, \Delta f=-22 \mathrm{~Hz},(\mathrm{~b}, \mathrm{c}, \mathrm{d}) 65 \times 136 \mathrm{~nm}^{2}, \Delta f \approx-22 ;-18 ;-20 \mathrm{~Hz}$, (e) $40 \times 40 \mathrm{~nm}^{2}, \Delta f=-48 \mathrm{~Hz}$, (f) $30 \times 26 \mathrm{~nm}{ }^{2}, \Delta f=-69 \mathrm{~Hz}$, All images: $f_{0}=275 \mathrm{kHz}, \mathrm{v}_{\mathrm{Scan}}=10 \mathrm{~Hz}(\mathrm{e}: 24 \mathrm{~Hz}, \mathrm{f}: 33 \mathrm{~Hz}), k=28 \mathrm{~N} / \mathrm{m}, \mathrm{A}_{\mathrm{pp}}=15 \mathrm{~nm}$. 


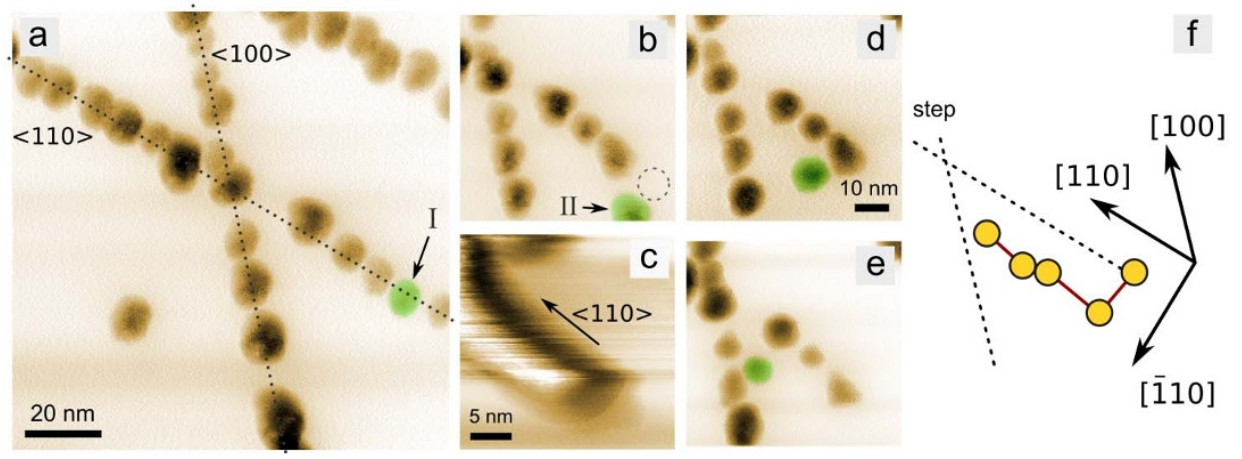

Figure 3 Experimental constant height images presenting several manipulation steps of Au clusters on $\mathrm{NaCl}(001)$ (nominal thickness: 0.64 monolayers, flux: $1.0 \times 10^{13}$ atoms $/ \mathrm{cm}^{2} / \mathrm{s}$ ). (a) Decoration of $\mathrm{NaCl}$ steps by Au clusters (cluster-cluster distance: $8.4 \mathrm{~nm}$, cluster density: $4.6 \times 10^{11}$ clusters $/ \mathrm{nm}^{2}$ ). (b) A single cluster (green) is separated from the step during a scan with the AFM. The dashed circle represents the position of the manipulated cluster in the previous image. (c) Controlled manipulation of the cluster by sliding. (d) The result after the movement. (e) Controlled manipulation of the cluster by kicking in a spectroscopy step. (f) Schematic of the manipulation steps. (a) $88 \times 88 \mathrm{~nm}{ }^{2}, \Delta f=-52 \mathrm{~Hz}$, (b, d, e) $54 \times 51 \mathrm{~nm}^{2}, \Delta f \approx-54 \mathrm{~Hz},(\mathrm{c}) 20 \times 20 \mathrm{~nm}^{2}, \Delta f=-120 \mathrm{~Hz}$, All images: $f_{0}=247 \mathrm{kHz}, k=32 \mathrm{~N} / \mathrm{m}, \mathrm{v}_{\mathrm{Scan}} \approx 15 \mathrm{~Hz}, \mathrm{~A}_{\mathrm{pp}}=14 \mathrm{~nm}$.

\section{Discussion}

The charged $\mathrm{Na}^{+}$and $\mathrm{Cl}^{-}$vacancies (latter are $\mathrm{F}^{+}$-centres) are expected to be mobile on the $\mathrm{NaCl}(001)$ surface and diffuse to steps during annealing ${ }^{35,36}$. Recent nc-AFM and Kelvin Probe Force Microscopy (KPFM) studies have further shown that $\mathrm{Na}^{+}$vacancies are the dominant defect at step-edges and kinks ${ }^{18,37}$, which is due to the Debye-Frenkel layer formation on alkali halide (001) surfaces ${ }^{38,39}$. This suggests that a cluster taken from a step, as shown in Fig. 3, is with a high probability anchored by a $\mathrm{Na}^{+}$vacancy. The experimentally observed kinetic anisotropy of these clusters can then be explained as the characteristic behaviour of this particular clusterdefect complex, as revealed by the simulations. The third type of possible defect on $\mathrm{NaCl}(001)$ is the $\mathrm{F}^{0}$-center $\left(\mathrm{Cl}^{-}\right.$vacancy + electron, i.e., $\mathrm{a} \mathrm{Cl}^{0}$ vacancy), and these defects are expected to be stable on the terrace (calculated diffusion barrier for the defect is $0.9 \mathrm{eV}$ ). Therefore, they are also the most probable defect on which clusters found on terraces are adsorbed (as in Fig. 2). From this consideration, the theoretically predicted small diffusion anisotropy for a cluster adsorbed on a $\mathrm{Cl}$ vacancy agrees with the experimentally found isotropic movement of the cluster on the terrace.

The reason why the defects influence the movement of clusters differently can be understood by examining the calculated structures of the bonded cluster-vacancy pairs. In Fig. 5 (a) and (b), the differential charge densities are shown for clusters in the presence of $\mathrm{Cl}$ and $\mathrm{Na}$ vacancies, respectively. This density represents the change in electronic charge upon bringing the cluster and the surface together ${ }^{40}$. In the presence of a $\mathrm{Cl}$ vacancy, the $\mathrm{Au}$ cluster bonds almost exclusively at one of its corners, attaching to a $\mathrm{Cl}$ ion next to the vacancy. In fact, the cluster tilts $10^{\circ}$ as the bonded corner is attracted to the $\mathrm{Cl}$ ion. Because of this, the cluster prefers to move by pivoting around the bonded corner, as schematically shown in Fig. 5 (c) (although the preference is not strong, as shown by the almost isotropic barriers in Fig. 4 (c)). Simultaneously, the $\mathrm{Cl}$ vacancy jumps to a neighboring site, allowing the cluster to bond to another corner. Combining these rotations leads to movement in the $\langle 100\rangle$ direction, but since the cluster is constantly reorienting itself, on average none of the $\langle 100\rangle$ surface directions is preferred and the cluster may in fact move in any direction by taking a zig-zag path.

On the other hand, if the cluster attaches to a $\mathrm{Na}$ vacancy, it does not bond at a corner, but along an edge which orients itself with an entire row of $\mathrm{Cl}$ ions on the surface. This is seen in Fig. 5 (b). Again, the cluster is seen to slightly tilt $\left(5.1^{\circ}\right)$ towards the active edge to facilitate the bonding. For such a configuration, the energetically favored mode of movement of the cluster is sliding along the row of $\mathrm{Cl}$ ions with which it has bonded, so that the active edge always stays on the $\mathrm{Cl}$ row. This is represented in Fig. 5 (d). Since this movement follows a single row of $\mathrm{Cl}$ ions, the cluster moves in a [110] direction. Notably, since the cluster cannot move perpendicularly to the $\mathrm{Cl}$ row without detaching or rotating first, a much more difficult task than sliding in the preferred direction, this configuration results in truly anisotropic movement. Exactly the same kind of attaching to a $\mathrm{Cl}$ row occurs with a larger cluster as shown in Fig. 5 (f), demonstrating that this behaviour does not depend on the cluster size.

There is a discrepancy between the cluster sizes in the presented theory and experiments (100 and 1000 atom clusters, respectively). Although such a difference in the number of atoms only means a linear size difference by a factor of two, difference in size at this scale may lead to changes in the cluster morphology. On ionic surfaces like $\mathrm{NaCl}(001)$ or $\mathrm{MgO}(001)$, gold clusters expose either their (001) or (111) facets to form rectangular or triangular shapes, respectively, and the shape may depend on the size and growth conditions $s^{44,45}$. However, as our simulations predict the anisotropic movement for the cluster-vacancy complex to be due to bonding of low-coordinated gold atoms at the cluster edge with $\mathrm{Cl}$ ions on the substrate near a Na vacancy, the anisotropy should not depend on the size and exact shape of the cluster. Instead, the behaviour is a characteristic feature of this kind of cluster-defect pair. We therefore expect the same characteristics also for the larger clusters, as seen in the experiments.

To summarize, we experimentally demonstrate nanomanipulation of gold clusters roughly of the size of 2000 atoms $(5 \mathrm{~nm}$ diameter) as single units on the terraces of the insulating $\mathrm{NaCl}(001)$ surface using nc-AFM. From first principles calculations we show that stable clusters on the terrace are expected to be adsorbed at defects, which always accompany a cluster during its movement on the surface. Since the $\mathrm{NaCl}(001)$ surface only has vacancies as potential defect candidates at the surface, the manipulation properties are dominated by the type of vacancy: clusters on cation vacancies exhibit an anisotropic movement along the $\langle 110\rangle$ surface directions whereas clusters on anion vacancies do not. Especially with respect to the cation vacancies, which are known to be located at the step edges, theory perfectly agrees with experiments since clusters that are detached from steps by the tip move indeed along $\langle 110\rangle$ surface directions.

In general, this work demonstrates both experimentally and theoretically that even on smooth surfaces cluster-defect pairs can have extremely anisotropic movement patterns, and that this anisotropy can be strong enough to be the dominant factor controlling their manipulation. Such constrained movement can be exploited when building structures through nanomanipulation or even in the design 
a
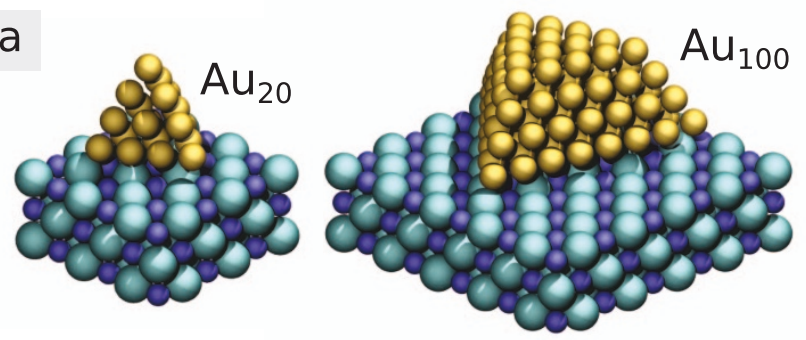

b

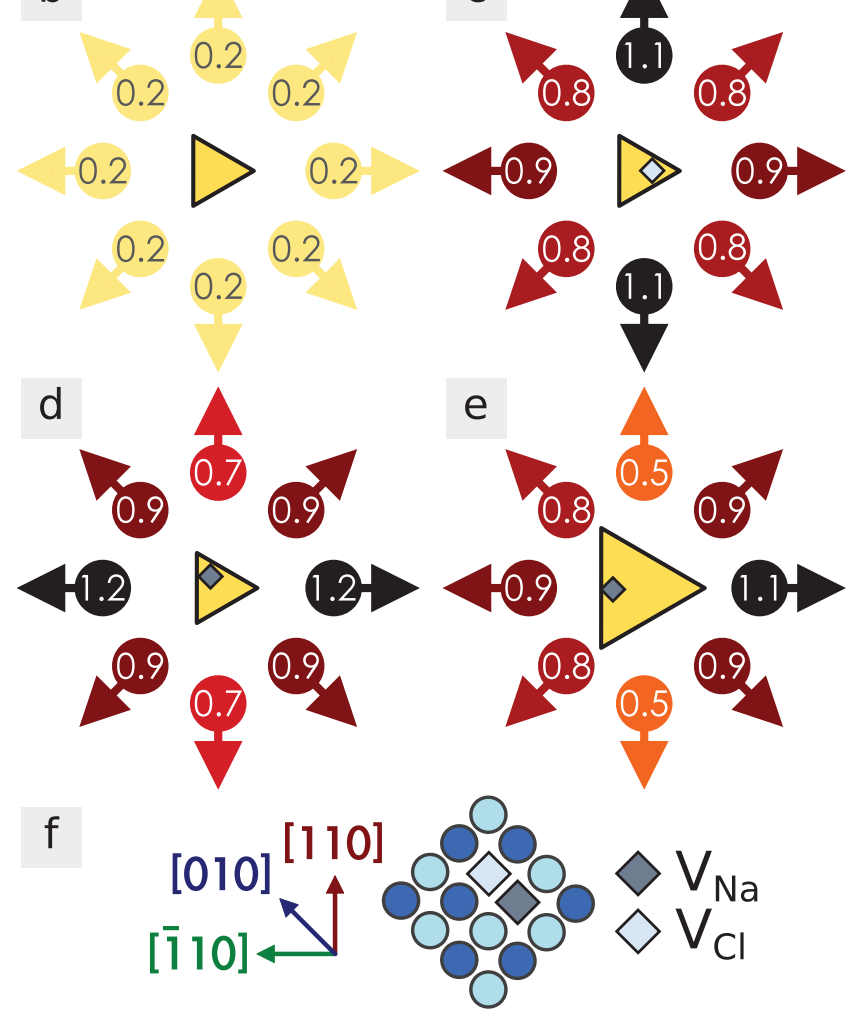

Figure 4 Calculated diffusion barriers for various cluster-vacancy structures in $\mathrm{eV}$. (a) The $\mathrm{Au}_{20}$ and $\mathrm{Au}_{100}$ clusters used in calculations. (b) $\mathrm{Au}_{20}$ without defect (c) $\mathrm{Au}_{20}$ on a Cl vacancy (d) $\mathrm{Au}_{20}$ on a Na vacancy (e) $\mathrm{Au}_{100}$ on a Na vacancy (vacancy at the energetically favoured edge site). (f) The $\langle 110\rangle$ directions are set to run horizontally and vertically in the figures. Positions of $\mathrm{Na}$ and $\mathrm{Cl}$ vacancies $\left(\mathrm{V}_{\mathrm{Na}}\right.$ and $\left.\mathrm{V}_{\mathrm{Cl}}\right)$ are denoted by dark and light squares, respectively.

of nanomechanical machines. Also conversely, observing specific anisotropy patterns could be used for distinguishing the types of defects on which clusters are adsorbed. Of course, this is not necessarily a one-to-one mapping: in a system where different kinds of defects exist, it is possible that different cluster-defect complexes have very similar manipulation patterns. Still, examining the cluster movement may allow one to distinguish the most likely defect types, especially if the intrinsic occurrence probabilities of defects are known.

Aside from providing an important characterization of defects, the ability to control and create defect-cluster complexes offers great potential for future studies in catalysis where, for instance, the cluster reactivity must be studied as a function of adsorption site. Such manipulation experiments can be combined with a control over the defects, which can be created by e.g. the AFM tip followed by imaging and spectroscopy identification, or by doping or irradiation of the substrate material such that known defects appear on the surface. When manipulation can be used for an overall characterization of the defect-cluster system, it will be a powerful tool for linking
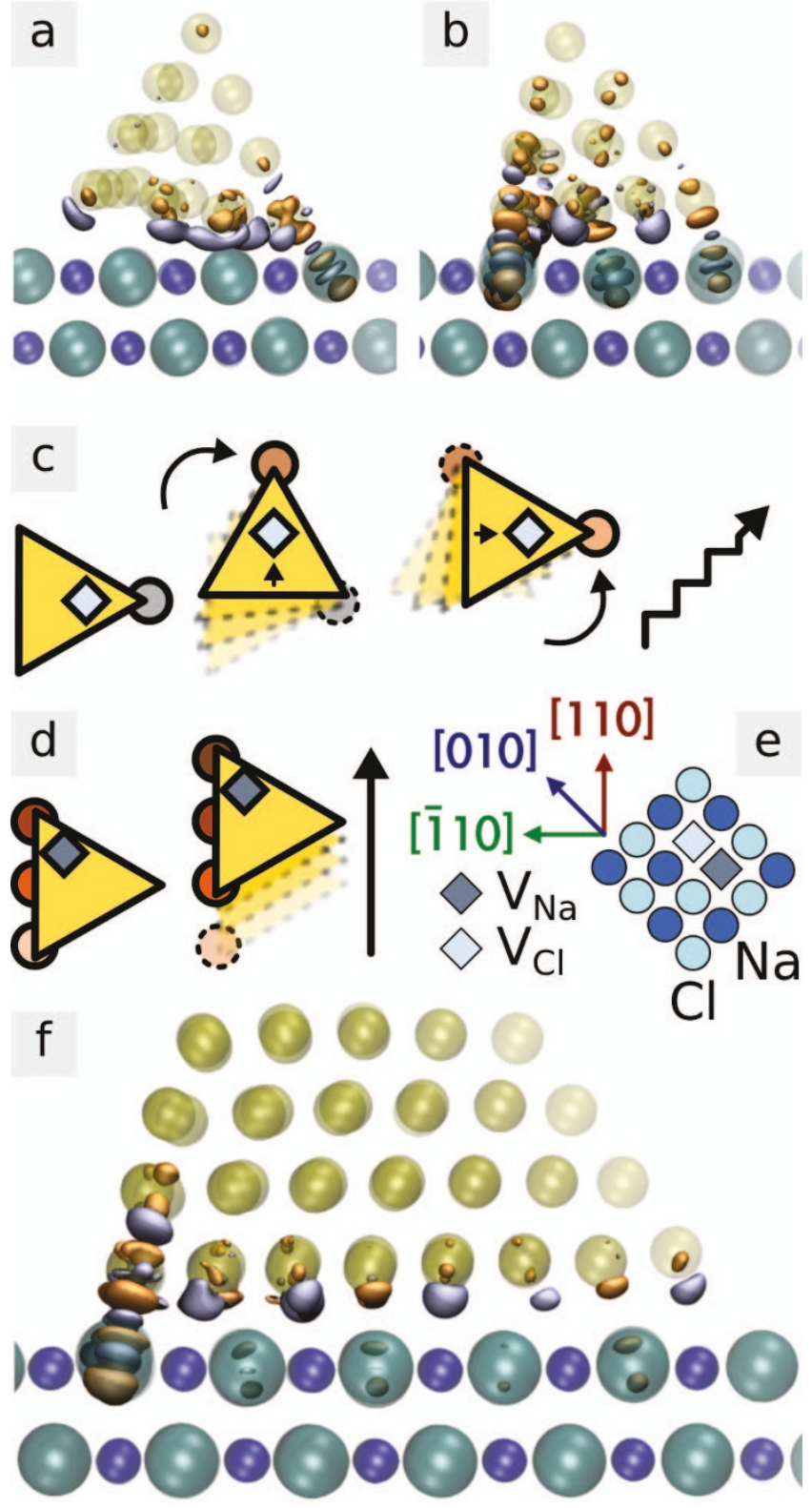

Figure $5 \mid$ Differential charge densities $\rho_{\mathrm{Au}+\mathrm{NaCl}}-\rho_{\mathrm{Au}}-\rho_{\mathrm{NaCl}}$ plotted at isosurfaces $-0.01 \mathrm{e}^{-3}$ (grey) and $0.01 \mathrm{e}^{-3}$ (orange) for $\mathrm{Au}_{20}$ on a $\mathrm{Cl}$ vacancy (a) and on a Na vacancy (b). The [110] direction runs into the image. Schematic representation of the preferred diffusion modes for the $\mathrm{Au}_{20}$ on a Cl vacancy (c) and a Na vacancy (d), with the [110] direction of the $\mathrm{NaCl}$ running vertically as shown in (e). Differential charge density for $\mathrm{Au}_{100}$ on a Na vacancy (f) at the same isosurfaces as in (a) and (b). Rendered using $\mathrm{VMD}^{57}$.

observed properties with the atomic and electronic structure of the defect-cluster complex.

\section{Methods}

Sample preparation. The undoped $\mathrm{NaCl}$ crystals are grown in an argon atmosphere by the Czochralski growth method in our laboratory at the $\mathrm{CINaM}^{41}$. Before surface preparation, crystals and sample holders are cleaned by annealing $\left(\sim 250^{\circ} \mathrm{C}\right)$ in an oven located inside the main UHV chamber ${ }^{42}$. After cooling, the (001) surfaces are prepared by cleavage of the crystals along the (001) plane at room temperature in the UHV chamber ${ }^{42}$. The crystals are then annealed a second time in the same oven at $\sim 150^{\circ} \mathrm{C}$ for a few hours to remove surface charges ${ }^{43}$. The preparation of a clean $\mathrm{NaCl}(001)$ surface by cleavage and following annealing, the deposition of gold onto such a $\mathrm{NaCl}(001)$ surface, and following AFM experiments are done in one UHV chamber, which is equipped with a sample transfer system. 
Gold coverages were estimated by measuring the flux of a beam of neutral gold atoms from a Knudsen cell using a quartz crystal microbalance. The pressure during the deposition is in the lower $10^{-9} \mathrm{mbar}$ range. The nc-AFM images were not used for coverage estimation due to the strong tip-cluster convolution, which is of importance when the clusters have a size of only a few nanometers ${ }^{15,16}$. Especially for the small clusters studied in this work, their size (diameter $\sim 5 \mathrm{~nm}$ ) is smaller than the tip apex, the diameter of which is typically $\leq 20 \mathrm{~nm}$ for new unused tips ${ }^{46}$. In this case, the shape of the tip apex is basically imaged at each cluster site, which becomes immediately evident when the apex changes upon tip changes during imaging. The convolution therefore overestimates the cluster size and induces large errors in any kind of estimates where the cluster size is a parameter.

AFM and manipulation protocols. Frequency-modulated noncontact AFM (ncAFM) and Kelvin probe force microscopy (KPFM) experiments are performed in the low $10^{-10} \mathrm{mbar}$ pressure range and at room temperature with an Omicron RT-AFM/ STM. Conducting silicon cantilevers are used (Nanosensors, p-Si, $0.015 \Omega . c m, 247$ and $275 \mathrm{kHz}$ resonance frequency, $32(247 \mathrm{kHz}$ tip) and $28 \mathrm{~N} / \mathrm{m}(275 \mathrm{kHz}$ tip) spring constant). Noncontact AFM primarily measures the change of frequency (detuning $\Delta f$ ) of the cantilever oscillation that is due to the interaction of the attached sensing tip with the surface ${ }^{17,47}$. In standard constant $\Delta$ foperation mode, a high feedback gain of the distance regulation loop is used such that variations in $\Delta f$ from a pre-set value $\left(\Delta f_{\text {pre-set }}\right)$ are nullified by regulating the tip-surface distance (topography images). For imaging the surface in a quasi-constant height mode, which was the scanning mode used for this work, the gain is drastically lowered such that the tip follows only the mean inclination of the surface. In this mode, the tip-surface interaction is stored in the $\Delta f$ image. When scanning above clusters the tip is closest above the top facet of the clusters so that the tip-surface interaction is strongest (dark contrast $\equiv$ stronger interaction $\equiv$ more negative detuning $\Delta f$ ). More details about scanning modes with respect to clusters can be found in Refs. 15, 16. In order to measure the detuning, a demodulator from NanoSurf is used (EasyPLL). All images presented in this work were conducted in the constant height mode of the AFM Images were acquired with the Omicron SCALA system and analyzed with the Gwyddion software ${ }^{48}$.

Two methods are used to manipulate the clusters by nc-AFM. The first method consists of moving the cluster by pushing during a spectroscopy step (Fig. 1 (a)). The scanning is switched off, the tip is positioned in a specific spot located on or very close to the cluster and the tip-sample distance is finally decreased monotonously. At a specific tip-cluster distance, at which the tip apex is very close to the cluster, the cluster moves on the surface due to a sufficiently large lateral force between the foremost atoms of the tip and the cluster. In the second method the tip is scanned preferentially in the constant height mode ${ }^{14-16}$ above the cluster (Fig. 1 (b)). From image to image, the tip-surface distance is reduced by choosing more negative detuning values $\Delta f$. At a specific distance, the tip comes from the side and is sufficiently close to the cluster such that again a lateral force between both moves the cluster. This produces an elongated contrast of the cluster, as can be seen in Fig. 2(f) and Fig. 3(c). From this specific contrast it can be concluded that the cluster slides by some small distance in front of the tip - the tip images almost always the same part of the cluster such that the same contrast is reproduced in each scanning line.

Calculations. Calculations were performed using spin polarized density functional theory (DFT), as implemented in the periodic plane wave code VASP ${ }^{49,50}$. Generalized gradient functionals $(\mathrm{PBE})^{51}$ and the projector augmented wave method (PAW ${ }^{49,52}$ were applied. An energy cutoff of $300 \mathrm{eV}$ and a $2 \times 2 \times 1 k$-point mesh were found to converge all key parameters of the system. The $\mathrm{NaCl}(001)$ surface was modeled using slabs three ionic layers thick with the bottom layer frozen, separated by $2.5 \mathrm{~nm}$ of vacuum. Energy barriers were determined using the climbing image nudged elastic band method ${ }^{53}$, where typically three or five chain images were sufficient. For simplicity, we ignore the effect of the AFM tip and concentrate on examining the energy landscape as felt by the clusters on the $\mathrm{NaCl}(001)$ surface.

Dispersion forces were not explicitly included in the calculations, and although they may influence adsorption energies, their effect on the diffusion barriers is expected to be small ${ }^{5,55}$. In order to verify that this is the case, we calculated as a benchmark a series of diffusion pathways using both PBE and the vdW-DF functional ${ }^{56}$, the latter implementing van der Waals forces. We especially used the vdWDF functional to re-examine the diffusion pathways predicted by PBE to have the lowest and highest barrier energies for the $\mathrm{Au}_{20}$ cluster and the different defects. In all cases, the functional including dispersion forces yielded energy barriers close to that given by PBE, $0.0-0.2 \mathrm{eV}$ lower, and the hierarchy of energies was the same for both functionals.

Lateral forces needed to move the clusters were also estimated from the calculated trajectories. In general, in the preferred pathways where the energy barriers were found to be the lowest, also the lateral force on the cluster was low, in the range of $0.1-0.2 \mathrm{eV} / \AA$. On the high barrier trajectories also the lateral forces were higher, about $0.2-0.3 \mathrm{eV} / \AA$. That is, a direct correlation was seen between the energy barriers and lateral forces. We focus on the energy barriers in the discussion, since these are more accurately determined in the calculations.

The calculated results were obtained for a charge neutral system unless stated otherwise. This means that to create a Na vacancy, we remove a $\mathrm{Na}$ atom from the lattice leaving behind effectively a $\mathrm{Na}^{+}$vacancy and a missing electron. Similarly, a Cl vacancy is effectively a $\mathrm{Cl}^{-}$vacancy with an additional electron. In the case of the anion vacancy, an electron remains localized in the vacancy forming an $F^{\circ}$-centre. This is the expected form of defects at the surface, particularly with the abundance of electron sources (i.e. metal) in the system. However, it is also possible to change the number of electrons in the calculations to simulate $\mathrm{Na}^{+}$and $\mathrm{Cl}^{-}$vacancies. These systems are not charge neutral, but the excess is compensated by a background charge. Extensive tests were performed to study the influence of the charge state of the defects on the results. These calculations are discussed in the Supplementary information.

20 and 100 atom gold clusters were used in the simulations, constructed in the shape of a tetrahedral pyramid or a truncated pyramid, respectively, as shown in Fig. 4 (a). Much larger systems are not feasible at this level of accuracy. Gold clusters prefer to grow in (100) epitaxy but also in (111) epitaxy with a triangular or hexagonal shape ${ }^{1,44,45}$. For consistency, we focus on small $\mathrm{Au}_{20}$ and $\mathrm{Au}_{100}$ clusters in (111) epitaxy in the calculations. For $\mathrm{Au}_{100}$, our calculations show the (111) faceted truncated tetrahedron structure to be energetically preferred to the best (100) epitaxial geometry we could find by $2.5 \mathrm{eV}$.

1. Henry, C. R. Surface studies of supported model catalysts. Surf. Sci. Rep. 31, 231-325 (1998).

2. Libuda, J. \& Fruend, H.-J. Molecular beam experiments on model catalysts. Surf. Sci. Rep. 57, 157-298 (2005).

3. Heiz, U. \& Landman, U. Nanocatalysis Springer-Verlag, Berlin (2007).

4. Adams, D. M. et al. Charge transfer on the nanoscale: Current status. J. Phys. Chem. B 107, 6668-6697 (2003).

5. Dietzel, D. et al. Frictional Duality Observed during Nanoparticle Sliding. Phys. Rev. Lett. 101, 125505 (2008).

6. Guerra, R., Tartaglino, U., Vanossi, A. \& Tosatti, E. Ballistic nanofriction. Nat. Materials 9, 634-637 (2010).

7. Mougin, K. et al. Manipulation of gold nanoparticles: Influence of surface chemistry, temperature, and environment (vacuum versus ambient atmosphere). Langmuir 24, 1577-1581 (2008).

8. Eigler, D. \& Schweizer, E. Positioning single atoms with a scanning tunneling microscope. Nature 344, 524-526 (1990).

9. Giessibl, F. Advances in atomic force microscopy. Rev. Mod. Phys. 75, 949-983 (2003).

10. Custance, O., Perez, R. \& Morita, S. Atomic force microscopy as a tool for atom manipulation. Nat. Nanotechnol. 4, 803-810 (2009).

11. Bouju, X., Joachim, C. \& Girard, C. Moving gold atoms with an atomic-forcemicroscope tip: A study of dimer and trimer formation on $\mathrm{NaCl}(100)$. Phys. Rev. B 50, 7893-7902 (1994).

12. Trevethan, T., Watkins, M., Kantorovich, L. N. \& Shluger, A. Controlled manipulation of atoms in insulating surfaces with the virtual atomic force microscope. Phys. Rev. Lett. 98, 028101 (2007).

13. Bartels, L., Meyer, G. \& Rieder, K. H. Basic steps of lateral manipulation of single atoms and diatomic clusters with a scanning tunneling microscope tip. Phys. Rev. Lett. 79, 679-700 (1997).

14. Barth, C., Foster, A. S., Reichling, M. \& Shluger, A. L. Contrast formation in atomic resolution scanning force microscopy on CaF2(111): experiment and theory. J. Phys.: Condens. Matter 13, 2061 (2001).

15. Barth, C., Pakarinen, O. H., Foster, A. S. \& Henry, C. R. Imaging nanoclusters in the constant height mode of the dynamic SFM. Nanotechnology 17, S128 (2006).

16. Pakarinen, O. H., Barth, C., Foster, A. S. \& Henry, C. R. Imaging the real shape of nanoclusters in scanning force microscopy. J. Appl. Phys. 103, 054313 (2008).

17. Barth, C., Foster, A. S., Henry, C. R. \& Shluger, A. L. Recent trends in surface characterization and chemistry with high-resolution scanning force methods. Adv. Mater. 23, 477-501 (2011).

18. Barth, C. \& Henry, C. R. Surface double layer on (001) surfaces of alkali halide crystals: A scanning force microscopy study. Phys. Rev. Lett. 98, 136804 (2007).

19. Hoffmann, R., Kantorovich, L., Baratoff, A., Hug, H. \& Guntherodt, H. Sublattice identification in scanning force microscopy on alkali halide surfaces. Phys. Rev. Lett. 92, 146103 (2004).

20. Foster, A. S., Barth, C. \& Henry, C. R. Chemical identification of ions in doped $\mathrm{NaCl}$ by scanning force microscopy. Phys. Rev. Lett. 102, 256103 (2009).

21. Teobaldi, G. et al. Chemical resolution at ionic crystal surfaces using dynamic atomic force microscopy with metallic tips. Phys. Rev. Lett. 106, 216102 (2011).

22. Gross, L. et al. Measuring the charge state of an adatom with noncontact atomic force microscopy. Science 324, 1428-1431 (2009).

23. Nony, L. et al. Observation of individual molecules trapped on a nanostructured insulator. Nano Lett 4, 2185-2189 (2004).

24. Mohn, F. et al. Reversible bond formation in a gold-atom-organic-molecule complex as a molecular switch. Phys. Rev. Lett. 105, 266102 (2010).

25. Lämmle, K. et al. Unambiguous determination of the adsorption geometry of a metal-organic complex on a bulk insulator. Nano Lett. 10, 2965-2971 (2010).

26. Dienel, T., Loppacher, C., Mannsfeld, S. C. B., Forker, R. \& Fritz, T. Growth-modeinduced narrowing of optical spectra of an organic adlayer. Adv. Mater. 20, 959-963 (2008).

27. Steiner, P. et al. Atomic-scale friction on stepped surfaces of ionic crystals. Phys. Rev. Lett. 106, 186104 (2011).

28. Steiner, P., Roth, R., Gnecco, E., Baratoff, A. \& Meyer, E. Angular dependence of static and kinetic friction on alkali halide surfaces. Phys. Rev. B 82, 205417 (2010).

29. Nony, L., Foster, A. S., Bocquet, F. \& Loppacher, C. Understanding the atomicscale contrast in Kelvin probe force microscopy. Phys. Rev. Lett. 103, 036802 (2009). 
30. Glatzel, T. et al. Determination of effective tip geometries in Kelvin probe force microscopy on thin insulating films on metals. Nanotechnology 20, 264016 (2009)

31. Harding, J., Stoneham, A. \& Venables, J. Predicting nucleation and growth processes: Atomistic modeling of metal atoms on ionic substrates. Phys. Rev. B 57, 6715-6719 (1998).

32. Hakala, M. H., Pakarinen, O. H. \& Foster, A. S. First-principles study of adsorption, diffusion, and charge stability of metal adatoms on alkali halide surfaces. Phys. Rev. B 78, 045418 (2008).

33. McKenna, K., Trevethan, T. \& Shluger, A. Interplay between adsorbate diffusion and electron tunneling at an insulating surface. Phys. Rev. B 82, 085427 (2010).

34. Bethge, H. Electron microscopic studies of surface structures and some relations to surface phenomena. Surf. Sci. 3, 33-41 (1964).

35. Pakarinen, O., Barth, C., Foster, A. S., Nieminen, R. \& Henry, C. High-resolution scanning force microscopy of gold nanoclusters on the $\mathrm{KBr}(001)$ surface. Phys. Rev. B 73, 235428 (2006).

36. Foster, A. S., Trevethan, T. \& Shluger, A. L. Structure and diffusion of intrinsic defects, adsorbed hydrogen, and water molecules at the surface of alkali-earth fluorides calculated using density functional theory. Phys. Rev. B 80, 115421 (2009).

37. Egberts, P., Filleter, T. \& Bennewitz, R. A kelvin probe force microscopy of charged indentation-induced dislocation structures in KBr. Nanotechnology 20, 264005 (2009)

38. Kliewer, K. \& Koehler, J. Space Charge in Ionic Crystals. I. General Approach with Application to NaCl. Phys. Rev. 140, A1226-A1240 (1965).

39. Kliewer, K. Space Charge in Ionic Crystals. II. The Electron Affinity and Impurity Accumulation. Phys. Rev. 140, A1241-A1246 (1965).

40. Molina, L. M. \& Hammer, B. Theoretical study of CO oxidation on Au nanoparticles supported by $\mathrm{MgO}(100)$. Phys. Rev. B 69, 155424 (2004).

41. Grange, G. Formation of the suzuki phase on the solid-liquid interface of czochralski grown crystals. Surf. Sci. 105, 265 (1981).

42. Barth, C., Claeys, C. \& Henry, C. R. Surface preparation of hard ionic crystals by ultrahigh vacuum cleavage. Rev. Sci. Instrum. 76, 083907 (2005).

43. Barth, C. \& Henry, C. R. Kelvin probe force microscopy on surfaces of UHV cleaved ionic crystals. Nanotechnology 17, S155-S161 (2006).

44. Henry, C. R. Morphology of supported nanoparticles. Prog. Surf. Sci. 80, 92-116 (2005).

45. Matthews, J. W. \& Grunbaum, E. The structure of gold films grown in ultra-high vacuum on sodium chloride substrates. Phil. Mag. 11, 1233-1244 (1965).

46. Nanosensors Neuchatel (Switzerland) http://www.nanosensors.com (accessed Jan 11th 2013).

47. Morita, S., Wiesendanger, R. \& Meyer, E. Noncontact Atomic Force Microscopy Springer, Berlin (2002)

48. Gwyddion http://gwyddion.net (accessed Jan 11th 2013).

49. Kresse, G. \& Joubert, D. From ultrasoft pseudopotentials to the projector augmented-wave method. Phys. Rev. B 59, 1758-1775 (1999).
50. Kresse, G. \& Furthmuller, J. Efficient iterative schemes for ab initio total-energy calculations using a plane-wave basis set. Phys. Rev. B 54, 11169-11186 (1996).

51. Perdew, J. P., Burke, K. \& Ernzerhof, M. Generalized gradient approximation made simple. Phys. Rev. Lett. 77, 3865-3868 (1996).

52. Blochl, P. E. Projector augmented-wave method. Phys. Rev. B 50, 17953-17979 (1994).

53. Henkelman, G., Uberuaga, B. \& Jonsson, H. A climbing image nudged elastic band method for finding saddle points and minimum energy paths. J. Chem. Phys. 113, 9901-9904 (2000)

54. Pakarinen, O. H. et al. Role of van der Waals forces in the adsorption and diffusion of organic molecules on an insulating surface. Phys. Rev. B 80, 085401 (2009).

55. Mura, M., Gulans, A., Thonhauser, T. \& Kantorovich, L. Role of van der Waals interaction in forming molecule-metal junctions: flat organic molecules on the Au(111) surface. Phys. Chem. Chem. Phys. 12, 4759 (2010).

56. Gulans, A., Puska, M. \& Nieminen, R. Linear-scaling self-consistent implementation of the van der Waals density functional. Phys. Rev. B 79, 201105 (2009).

57. Humphrey, W., Dalke, A. \& Schulten, K. VMD: Visual molecular dynamics. J. Mol. Graphics 14, 33 (1996).

\section{Acknowledgements}

We acknowledge financial support through the FANAS project NOMCIS. CB and GC highly acknowledge stimulating discussions with C. R. Henry. TH and ASF acknowledge support by the Academy of Finland through its Centres of Excellence Program (project no. 251748), as well as the computational resources offered by CSC, Finland.

\section{Author contributions}

All authors contributed to writing the article. CB and GC designed and performed experiments and analysis. TH and ASF designed the computational study. TH performed the calculations and analysis.

\section{Additional information}

Supplementary information accompanies this paper at http://www.nature.com/ scientificreports

Competing financial interests: The authors declare no competing financial interests.

License: This work is licensed under a Creative Commons

Attribution-NonCommercial-ShareALike 3.0 Unported License. To view a copy of this license, visit http://creativecommons.org/licenses/by-nc-sa/3.0/

How to cite this article: Hynninen, T., Cabailh, G., Foster, A.S. \& Barth, C. Defect mediated manipulation of nanoclusters on an insulator. Sci. Rep. 3, 1270; DOI:10.1038/srep01270 (2013) 